\section{Long-term Outcomes} of Fixed-Combination Halobetasol Propionate and Tazarotene Lotion Stratified by Baseline Signs and Symptoms of Psoriasis

\section{OBJECTIVE}

- In this post hoc analysis of a 52-week open-label study (NCT02462083),' we assessed the efficacy and safety of once-daily halobetasol propionate $(0.01 \%)$ and tazarotene $(0.045 \%)$ lotion (HP/TAZ) in 550 participants with psoriasis stratified by baseline signs and symptoms of disease

\section{CONCLUSIONS}

- Long-term use of HP/TAZ was generally associated with treatment success regardless of baseline symptom severity, and no new safety signals emerged symptom severity
over 52 weeks

- Participants with mild baseline symptoms were less likely to experience local skin reactions postbaseline compared with participants with more severe baseline symptoms

- Evaluation of patients' baseline itch, dryness, and stinging/burning may help predict outcomes of HP/TAZ treatment

- Clinicians can use this information to counsel patients regarding treatment expectations when initiating HP/TAZ

Leon Kircik,' Lawrence Green, ${ }^{2}$ Joshua Zeichner, ${ }^{3}$ Javier Alonso, ${ }^{4}$ Abby Jacobson ${ }^{5}$

Icahn School of Medicine at Mount Sinai, New York, NY; '²George Washington University School of Medicine, Washington, DC; ${ }^{3}$ Skin and Laser Center at Mount Sinai, New York, NY; 'Coral Gables Dermatology \& Aesthetics, Coral Gables, FL; 'SOrtho Dermatologics (a division of Bausch Health US, LLC), Bridgewater, NJ

\section{SYNOPSIS}

Topical psoriasis treatments may be used as
monotherapy for mild disease or as adjunct therapy

- Fixed-combination halobetasol propionate $(0.01 \%)$ and tazarotene $(0.045 \%)$ lotion (HP/TAZ) is
approved for treatment of plaque psoriasis in adults Given that patients' experiences with psoriasis differ
greatly further consideration and assessment of the greatly, further consideration and assessment of the
utility of HPITAZ in patients with varying symptom
severity is warranted

\section{METHODS}

All participants received once-daily HPITAZ for
8 weeks (Figure I)

8 weeks (Figure $)$

At week 8, participants who achieved the primary
endpoint of treatment success (defined as investigator's global assessment [IGA] of clear $[0]$ or almost clear [I]) stopped HP/TAZ and were throush 52 wecks Those who did noated as needed through 52 weeks. Those who did not achieve
treatment success at week 8 continued HPITAZ Participants were allowed 24 continuous weeks
of HPTTAZ treatment if they achieved $\geq 1$-grade of HP/TAZ treatment if they achieved $\geq 1$-grade
improvement in IGA from baseline at week 12 , with
monthly reevaluation for achevement of IGA $/ 1$. In this post hoc analysis, 550 participants were
stratified by baseline severity of itch, dryness, and stinging/burning (none to mild vs moderate to Itch and stinginglburning were scored on a scale from
0 (none) to 3 severere as reported by the participant
in the past 24 hours in the past 24 hours
Dryness was scored on a scale from 0 (none) to 3
(severe) as assessed by the investigator

Figure I. Design of the long-term open-label study
f

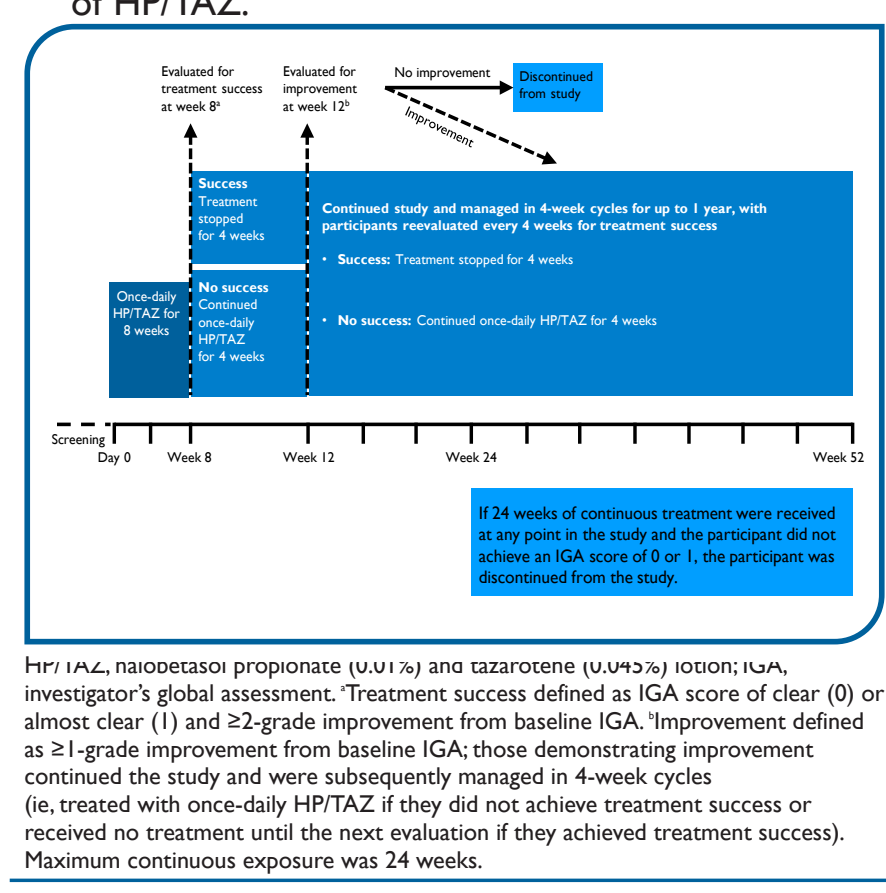

RESULTS

Participant population

- Baseline characteristics are shown in Table I

Table I. Participants Stratified by Baseline Severity of Signs/Symptoms

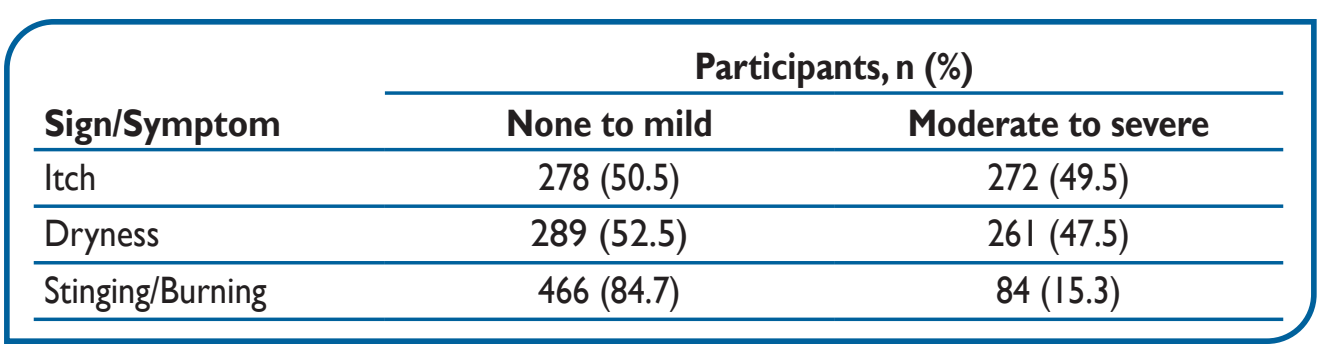

Efficacy

- At week 52 , a greater proportion of participants with none-to-mild baseline
signs/symmtoms had treatment success (IGA 0 or I) compared with participants

signs/symptoms had treatment success (IGA 0 or I) compare
with moderate-to-severe baseline signs/symptoms (Figure 2)

Figure 2. Treatment success at week 52 among participants treated with
HPITAZ stratified by baseline severity of itch, dryness, and stinginglburning

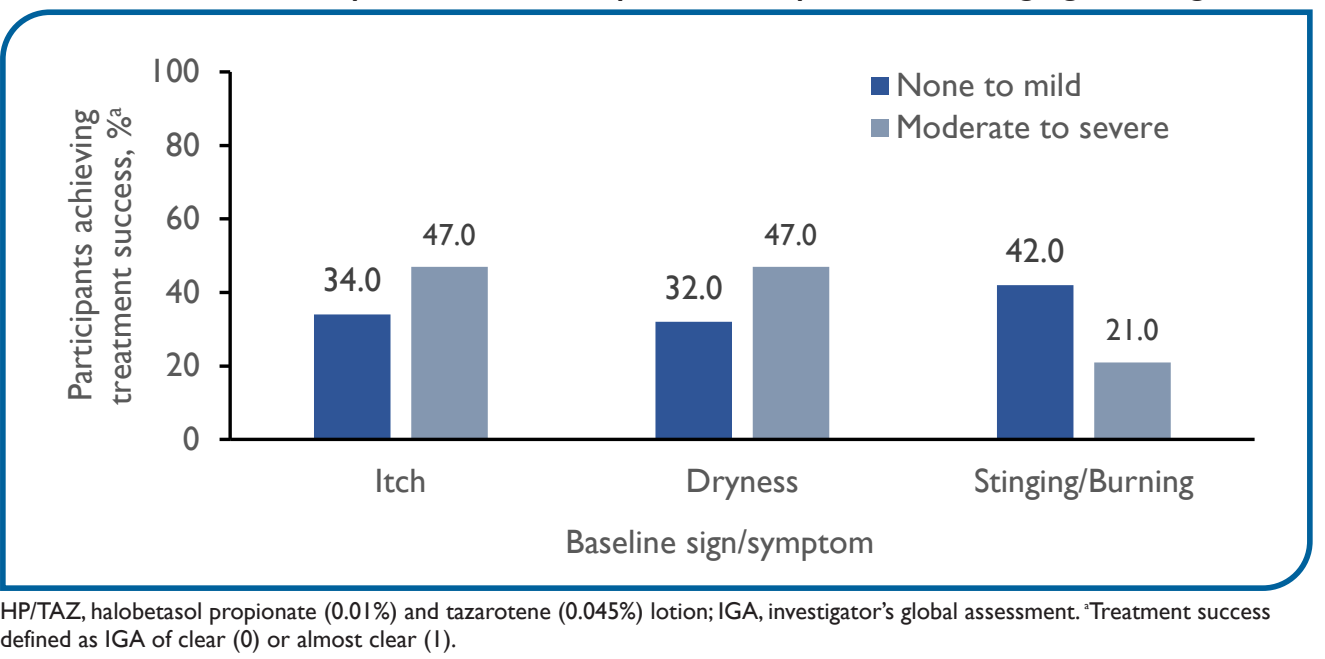

Participants with none-to-mild signs/symptoms at baseline were more likely to experience no signs/symptoms at any time point postbaseline relative to
participants with moderate-to-severe signs/symptoms at baseline (Figure 3)

Figure 3. Participants without signs/symptoms at any time point postbaseline
stratified by baseline severity of itch, dryness, and stinging/burning.

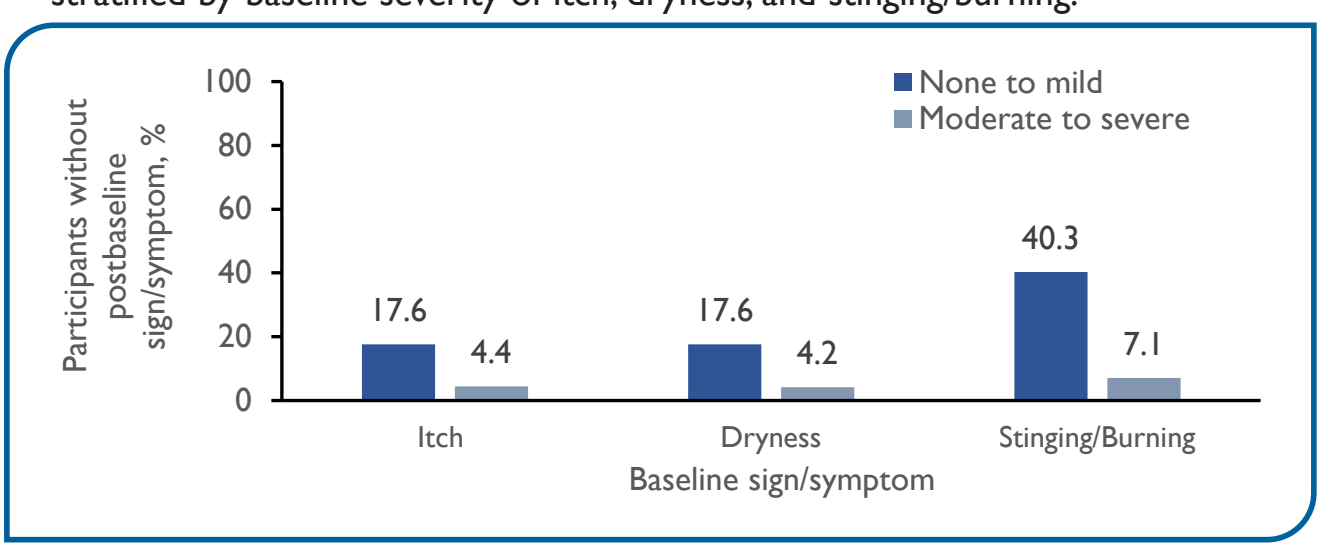

Participants with moderate-to-severe itch (Figure AA), dryness (Figure 4B), or burning/stinging (Figure 4 C) at baseline wer
to-severe posstbaseline local skin reactions
Figure 4. Of the participants who experienced moderate-to-severe
postbaseline signs/symptoms stratified by baseline severity of (A) itch, (B)

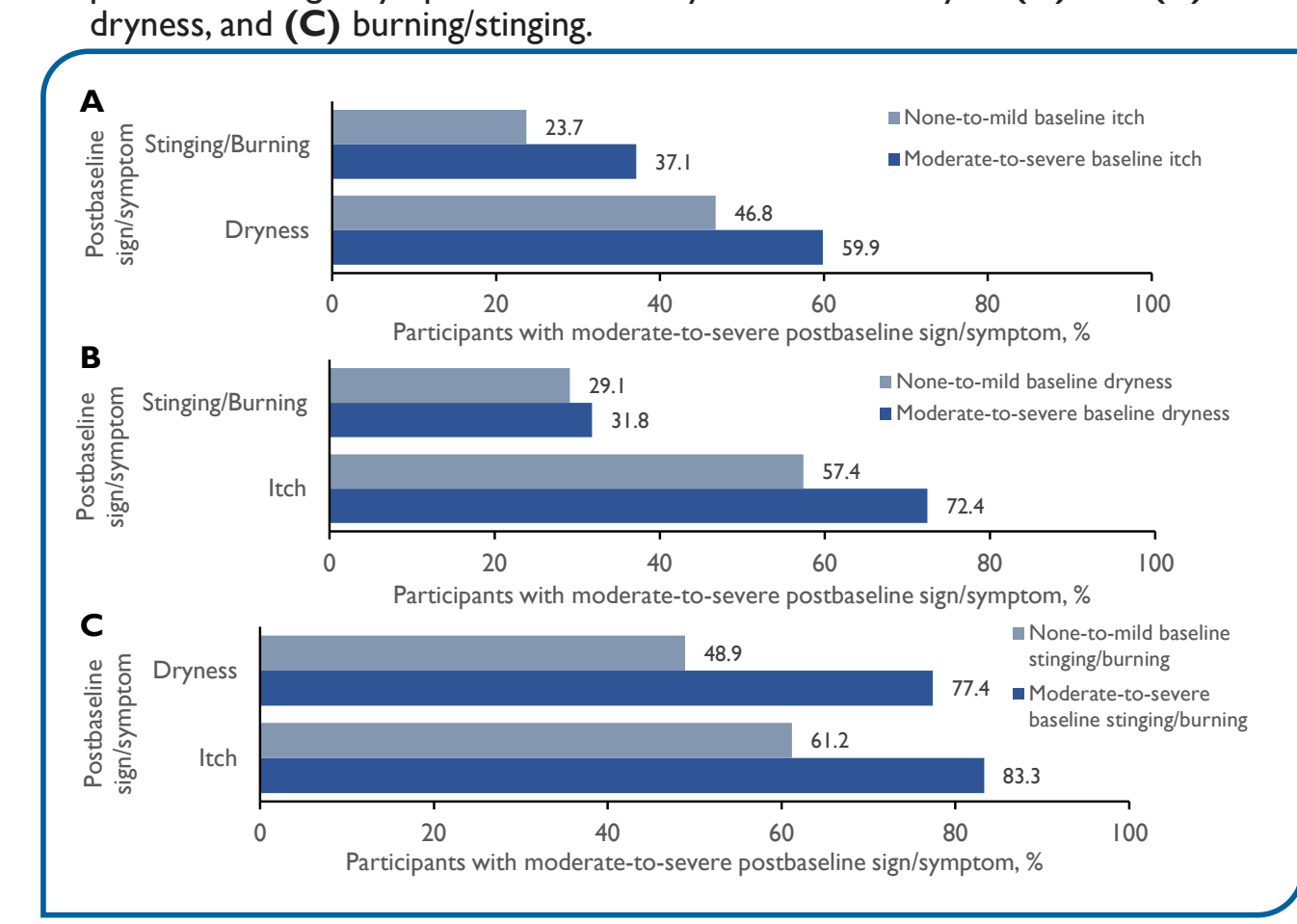

Safety

Rates of adverse events (AEs) were similar across groups and discontinuations
due to AEs were low (range, $5.6 \%-8.3 \%$ across baseline subgroups), similar to

what was seen in the overall population

Application site dermatitis was the most common treatment-emergent AE across
groups (Table 2)

Table 2. Treatment-Emergent Adverse Events Stratified by Baseline Severity of
Signs/Symptoms

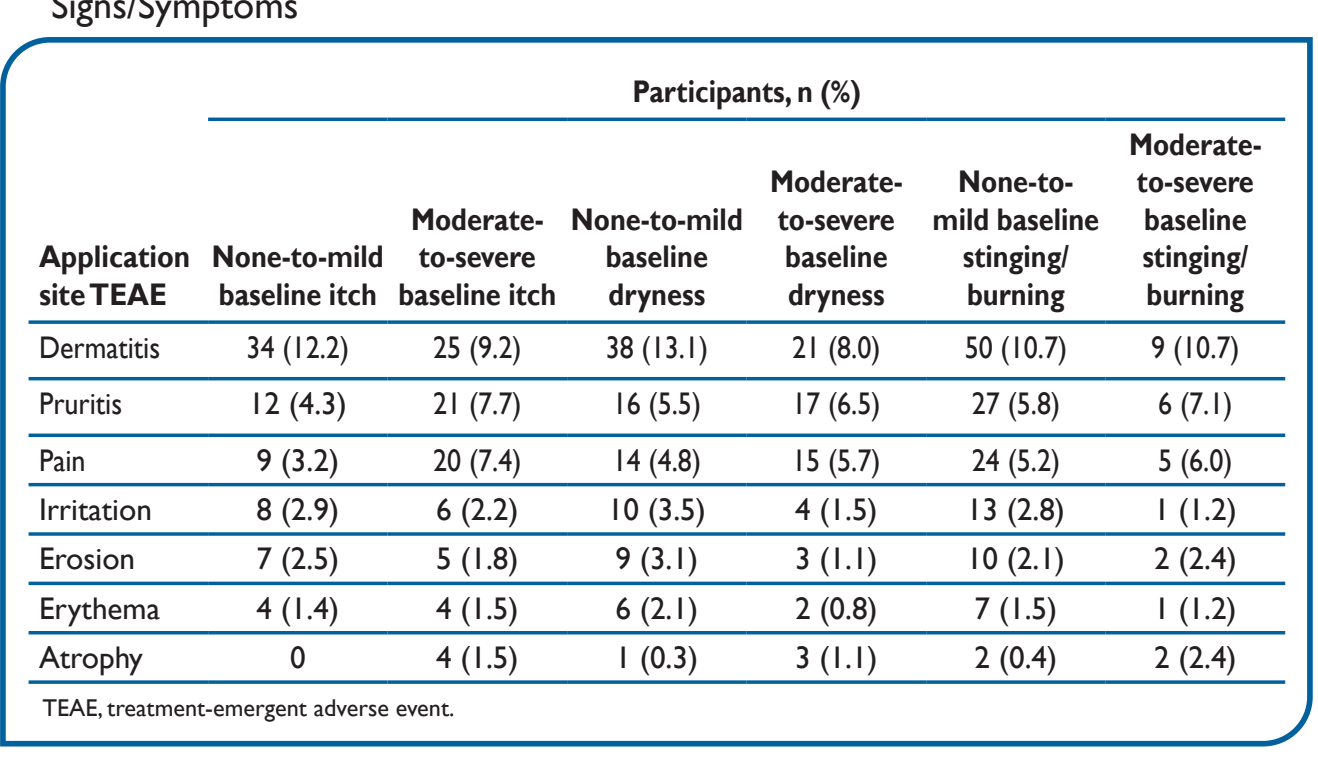

Acknowledgments: This study was sponsored by Ortho Dermatologics. Medical writing
support was provided by MedThink scicom and funded by Ortho Dermatoologics. Ortho
Dermatologics is d division of Bausch Health US.

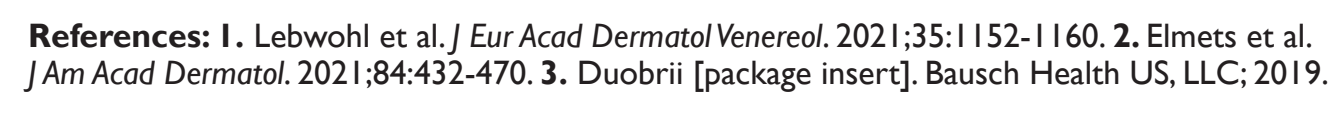

